\title{
Supporting Information: Influence of the Cation on the Surface Electronic Band Structure and Magnetic Properties of Mn:ZnS and Mn:CdS Quantum Dot Thin Films
}

Andrew J. Yost ${ }^{1,5^{*}}$, Thilini K. Ekanayaka ${ }^{1}$, Gautam Gurung ${ }^{1}$, Gaurab Rimal ${ }^{2}$, Sabit Horoz ${ }^{3}$, Jinke Tang $^{4}$, Tula Paudel ${ }^{1}$, and TeYu Chien ${ }^{4}$

${ }^{1}$ Department of Physics and Astronomy, University of Nebraska-Lincoln, Lincoln, NE 685880299, USA

${ }^{2}$ Department of Physics, Rutgers University, Piscataway, NJ 08854 USA

${ }^{3}$ Department of Electrical and Electronics Engineering, Sïrt University, Sïrt, Turkey

${ }^{4}$ Department of Physics and Astronomy, University of Wyoming, Laramie, WY 82071, USA

5Department of Physics, Oklahoma State University, Stillwater, OK 74075, USA

*Corresponding author: andrew.yost@okstate.edu

The following is supporting information containing fittings of XRD and XPS data from the main manuscript, and STM topography image, each figure caption contains a description of the figure. 

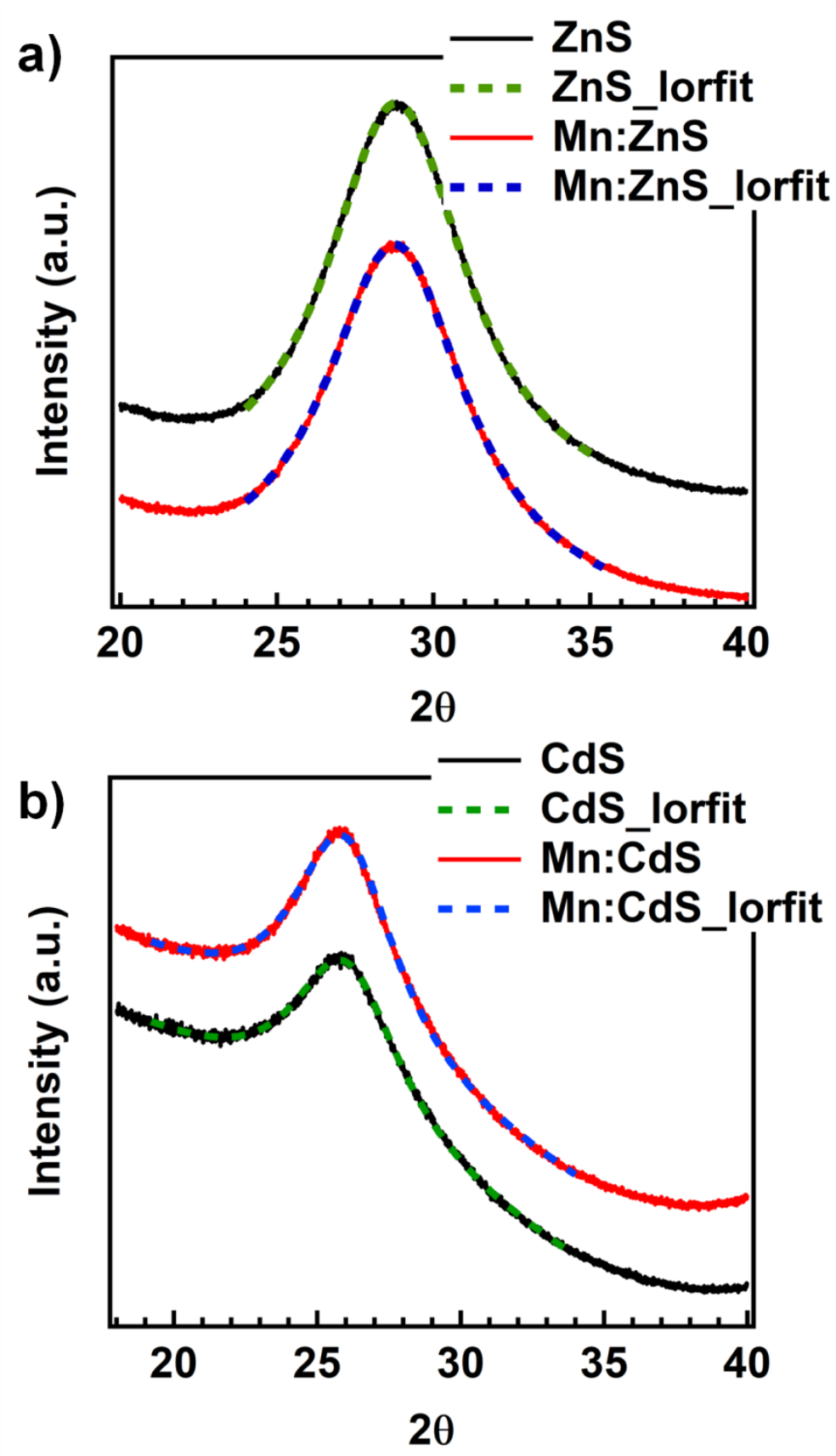

Figure S1: XRD for a) ZnS (111) peak with Lorentz function fitting (dashed lines) for both ZnS QD's (black and dashed green) and Mn:ZnS QD's (red and dashed blue) and b) $C d S$ (111) peak with Lorentz function fitting (dashed lines) for both CdS QD's (black and dashed green) and Mn:CdS QD's (red and dashed blue). 

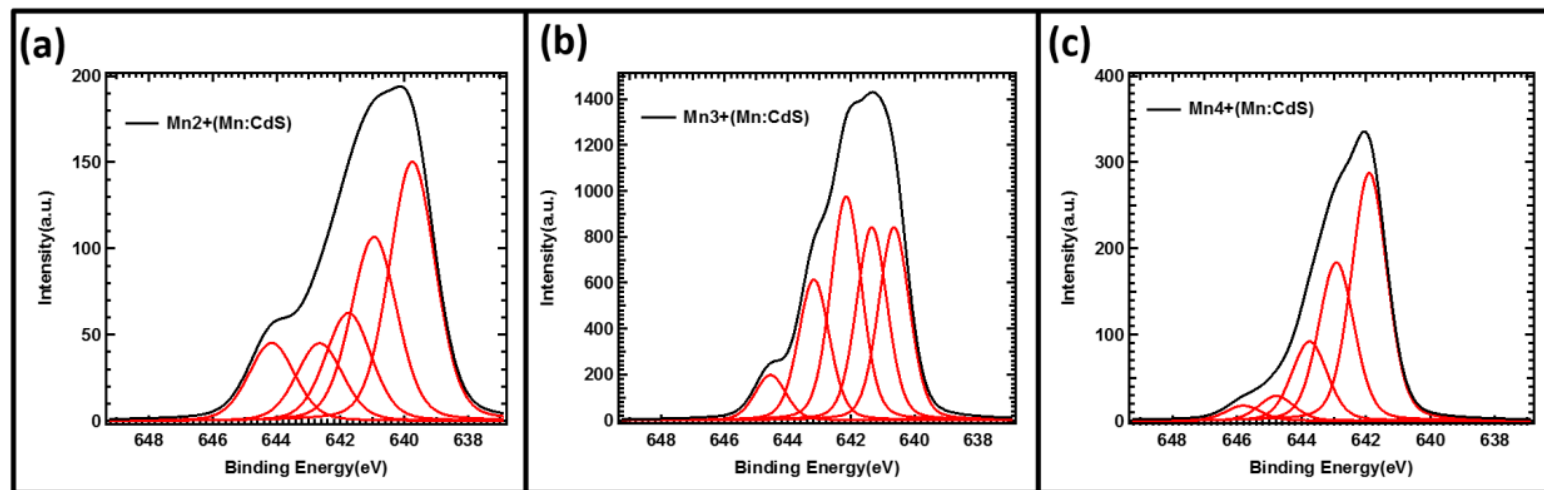

\begin{tabular}{|c|c|c|c|c|c|c|c|c|c|c|c|c|c|}
\hline Peak1 & ratio & Peak2 & ratio & Peak3 & ratio & Peak4 & ratio & Peak5 & ratio & FWHM(1-5) & Shake Up & Ratio & FWHM \\
\hline \multicolumn{14}{|l|}{$\mathrm{Mn} 2+$} \\
\hline 639.75 & 1 & 640.95 & 0.71 & 641.75 & 0.4167 & 642.65 & 0.3 & 644.15 & 0.3 & 1.7 & 646 & 0.303 & 2.5 \\
\hline \multicolumn{14}{|l|}{$\mathrm{Mn3+}$} \\
\hline 640.65 & 1 & 641.35 & 1 & 642.16 & 1.16 & 643.18 & 0.73 & 644.55 & 0.28 & 1.15 & & & \\
\hline \multicolumn{14}{|l|}{$\mathrm{Mn} 4+$} \\
\hline 641.9 & 1 & 642.92 & 0.64 & 643.75 & 0.32 & 644.78 & 0.1 & 645.8 & 0.06 & 1.3 & & & \\
\hline
\end{tabular}

Figure S2. Mn:ZnS QD's XPS Mn oxidation state envelope fitting peak (black) and multiplet fitting peaks(red peaks) for (a) Mn 2+, (b) Mn 3+, and (c) Mn 4+. (d) is the table of fitting parameters used for each multiplet fitting in $(a),(b), \&(c)$. 

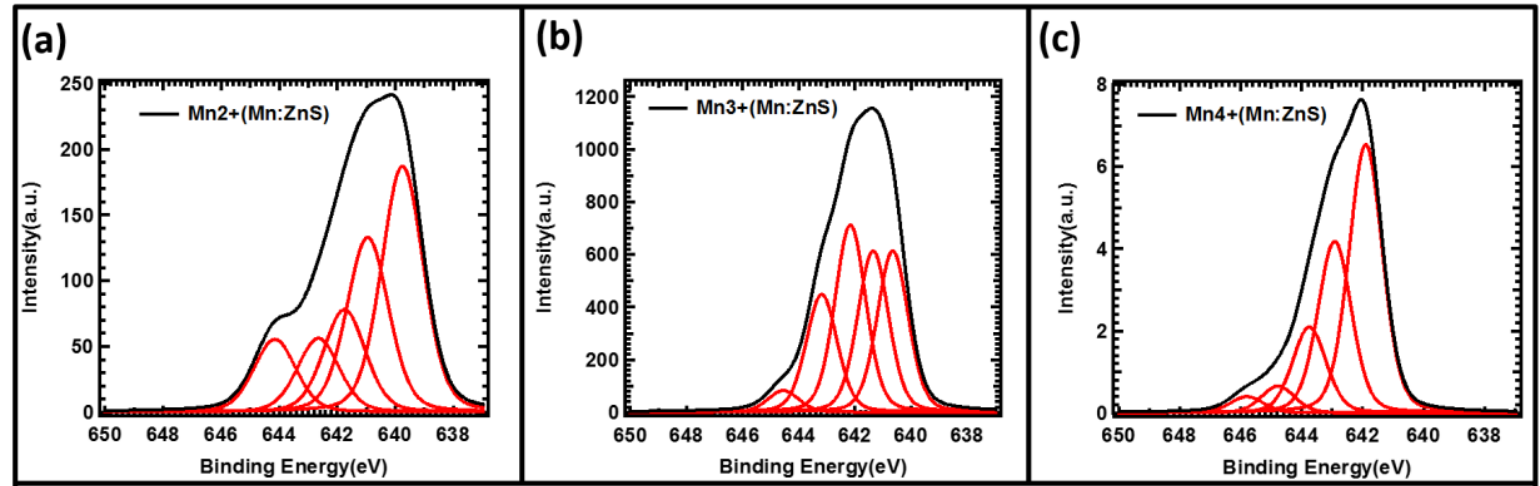

\begin{tabular}{|c|c|c|c|c|c|c|c|c|c|c|c|c|c|}
\hline Peak1 & ratio & Peak2 & ratio & Peak3 & ratio & Peak4 & ratio & Peak5 & ratio & FWHM(1-5) & Shake Up & Ratio & FWHM \\
\hline \multicolumn{14}{|l|}{$\mathrm{Mn2+}$} \\
\hline \multicolumn{14}{|l|}{ Mn3+ } \\
\hline 640.65 & 1 & 641.35 & 1 & 642.16 & 1.16 & 643.18 & 0.73 & 644.55 & 0.28 & 1.3 & & & \\
\hline 641.9 & 1 & 642.92 & 0.64 & 643.75 & 0.32 & 644.78 & 0.1 & 645.8 & 0.06 & 1.3 & & & \\
\hline
\end{tabular}

Figure S3. Mn:CdS QD's XPS envelope fitting peak (black) and multiplet fitting peaks(red peaks) for (a) Mn 2+, (b) Mn 3+, and (c) Mn 4+. (d) is the table of fitting parameters used for each multiplet fitting in $(a),(b), \&(c)$. 


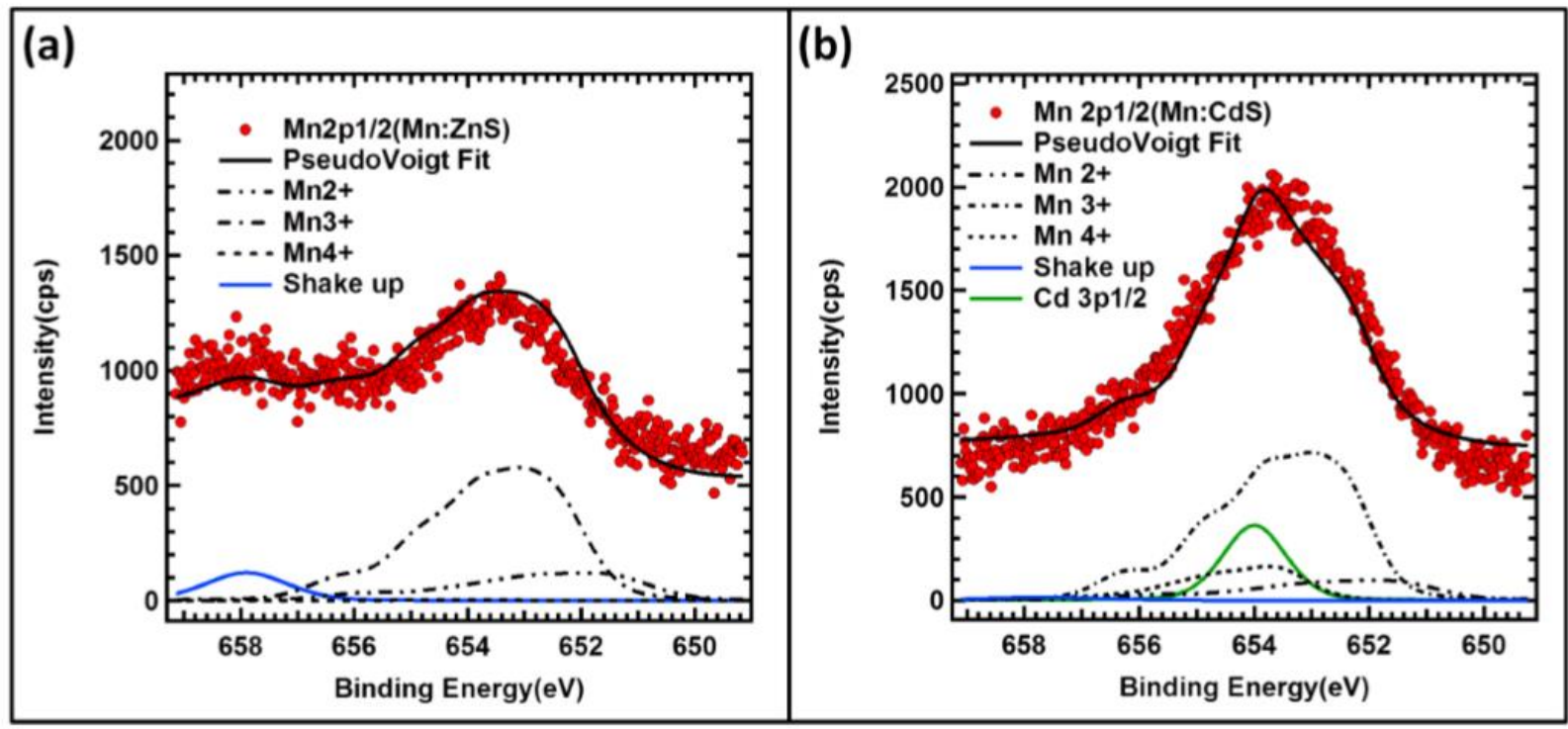

Figure S4. XPS spectra for (a) Mn 2p1/2 peak (red dots) in Mn:ZnS with Pseudo-Voigt fit(black line), below the data are the fitting curves of the envelope multiplet peaks for Mn $2+, 3+, \& 4+($ black dashed) along with the Shake up peak (blue). All peak fitting peak intensities are $1 / 2$ the intensity of the Mn 2p3/2 fitting peaks, (b) Mn 2p1/2 peak (red dots) in Mn:CdS with Pseudo-Voigt fit(black line), below the data are the fitting curves of the envelope multiplet peaks for Mn 2+, 3+, \& 4+(black dashed) along with the Shake up peak (blue). The dashed and blue fitting peak intensities are $1 / 2$ the intensity of the Mn $2 p 3 / 2$ fitting peaks. The green peak represents the Cd 3pl/2 peak which overlaps the Mn 2p1/2 

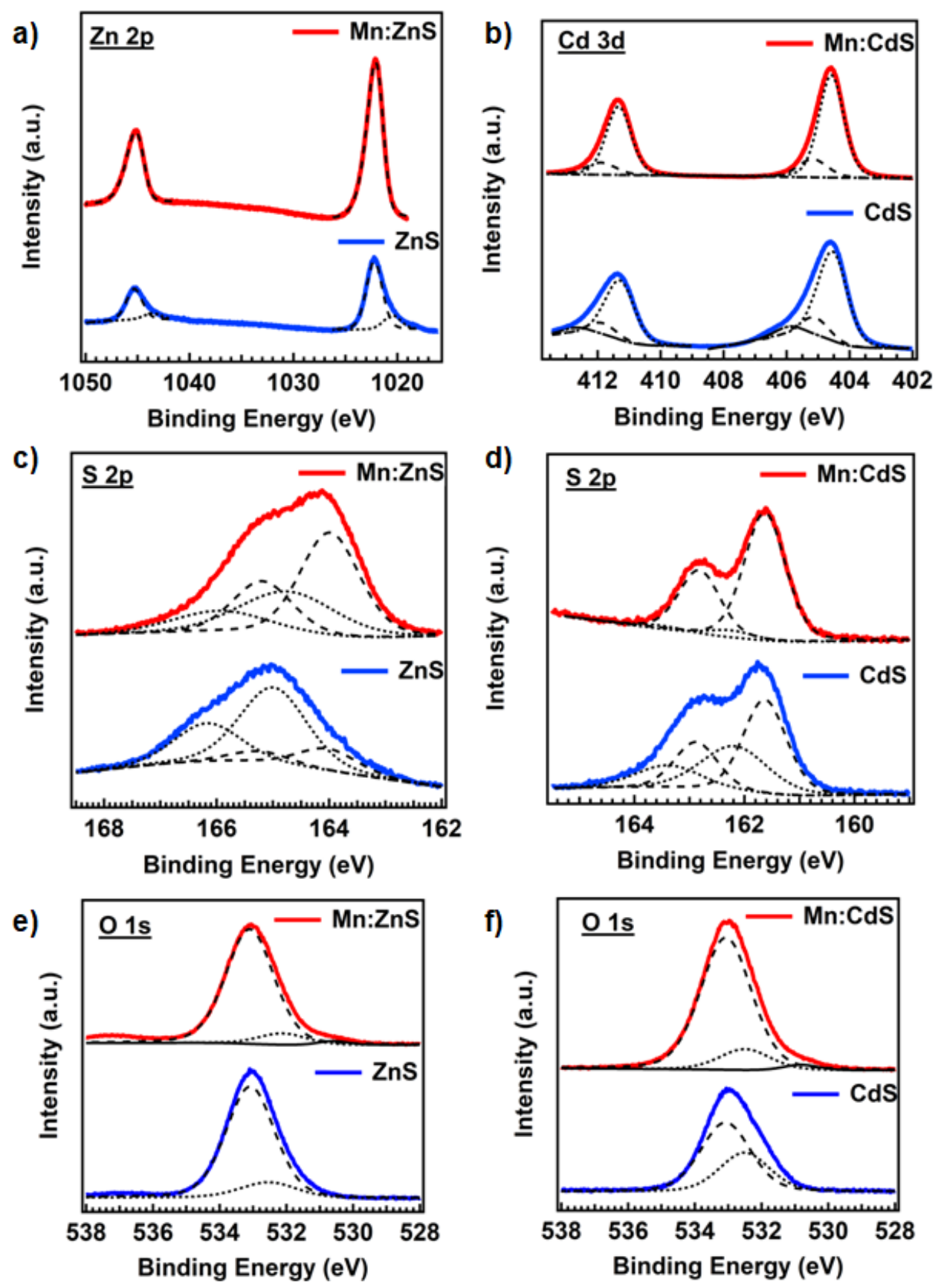

Figure S5: XPS spectra for (a) Zn 2p core level features in Mn doped (red curve) and undoped (blue curve) ZnS, peak fit curves indicate lower binding energy peak(1020 eV) due to sulfur vacancies in $\mathrm{ZnS}$, (b) Cd $3 d$ core level features in Mn doped (red curve) and undoped (blue curve) $C d S$, peak fit curves indicate lower binding energy peak $404 \mathrm{eV}$ ) due to sulfur vacancies in both Mn doped an d undoped CdS suggesting metallic Cd rests at the surface additionally there is a contribution from $\mathrm{Cd}^{2+}(405 \mathrm{eV})$, and a shake-up peak (406 eV), (c) and (d) $\mathrm{S} \mathrm{2p}$ core level features in Mn doped (red) and undoped(blue)for ZnS and CdS, respectively, the higher binding energy peaks are due to cation vacancies while the lower binding energy peaks are due to $S^{2-}$, and (e) and (f) $O 1$ s core level features in Mn doped (red) and undoped(blue) ZnS and CdS, respectively, There is a siginificant presence of $\mathrm{SiO}_{2}(533 \mathrm{eV})$ due to the substrate and a peak around $(532.5 \mathrm{eV})$ due to water in the films. Notice in the doped systems a negligible amount of $\mathrm{ZnO}_{2}$ and $\mathrm{CdO}_{2}$ is present as indicated by the lower binding energy peak around $531 \mathrm{eV}$. 

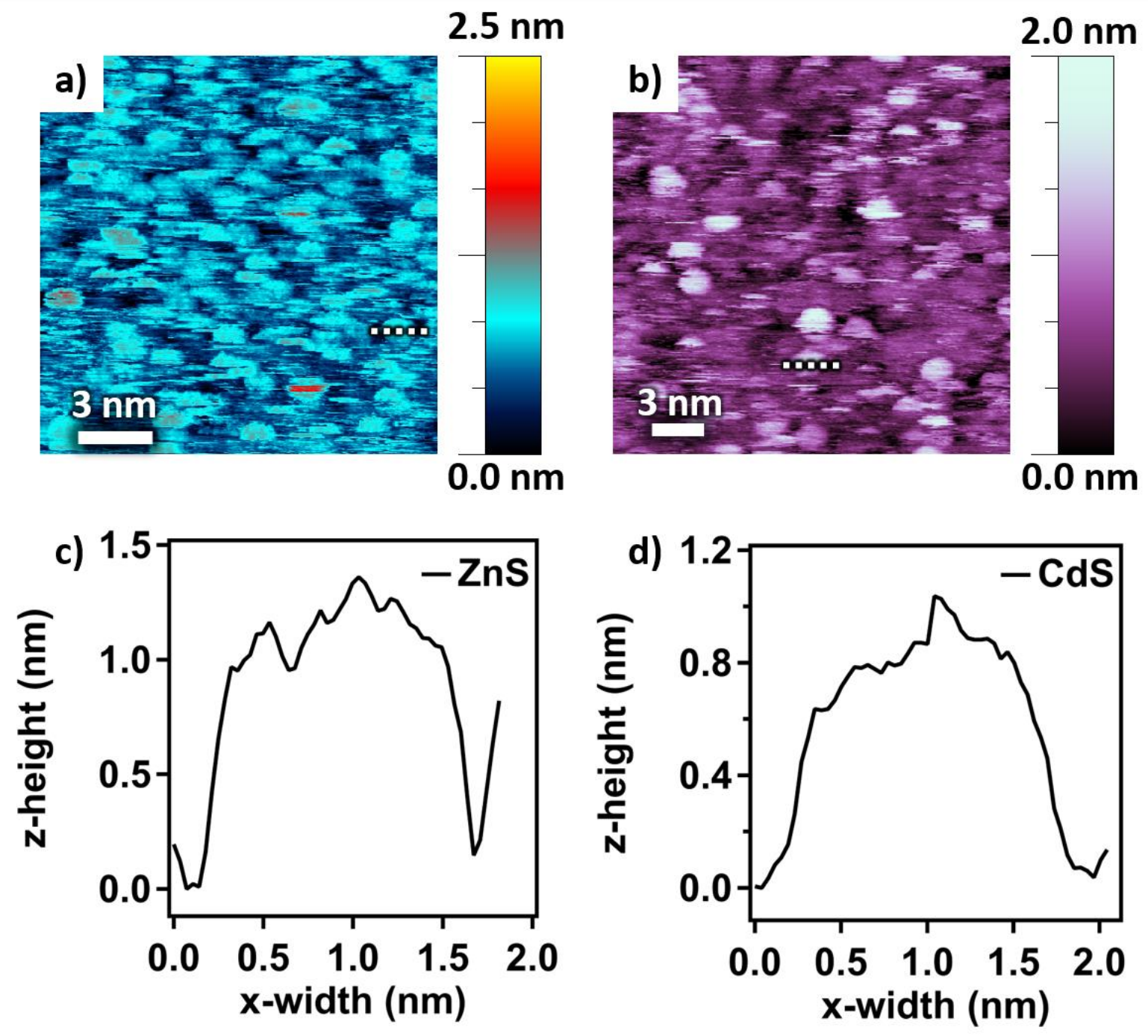

Figure S6: STM topography image of a) ZnS quantum dot thin film, image is $16.5 \times 16.5 \mathrm{~nm}^{2}$, $I_{\text {set }}=500 \mathrm{pA}$, bias $\left.=-3.0 \mathrm{~V}, \mathrm{~b}\right) \mathrm{CdS}$ quantum dot thin film, image is $23 \times 23 \mathrm{~nm}^{2}, I_{\text {set }}=300 \mathrm{pA}$, bias=-3.0 $\mathrm{V}, \mathrm{c}$ ) line profile of $\mathrm{ZnS}$ quantum dot taken along white dashed line in a), and d) line profile of CdS quantum dot taken along white dashed line in $b$ ). 